\title{
Dependence of sperm morphology and ejaculate characteristics on sperm concentration in the ejaculates of Hypor boars
}

\author{
Krzysztof Górski, Stanisław Kondracki, Anna Wysokińska, Maria Iwanina \\ Department of Animal Reproduction and Hygiene, Faculty of Natural Sciences, \\ Siedlce University of Natural Sciences and Humanities, 08-110 Siedlce, Poland \\ krzysztof.gorski@uph.edu.pl
}

Received: March 21, $2018 \quad$ Accepted: August 24, 2018

\begin{abstract}
Introduction: Determination of sperm concentration and morphology in ejaculate is essential in evaluating fertility. Sperm shape and dimensions may depend on their concentration in ejaculate. The aim of the study was to evaluate the dependence of Hypor boar morphological and morphometric sperm characteristics on concentration in ejaculate. Material and Methods: The study was conducted on 120 ejaculates from 12 Hypor boars. Depending on sperm concentration, they were put into three groups: low, medium, and high. Ejaculate sperm concentration was determined with the photometric method. Slides were prepared from semen samples which were evaluated for the morphology of spermatozoa. The preparations for morphological analyses were by the eosin-gentian staining method. Results: As the sperm concentration in the ejaculate increased, the length, perimeter, and area of the sperm heads also did. In the ejaculates with higher sperm concentration the sperm heads were rounder. The ratios of head dimensions to tail length or total length were the highest in the sperm from ejaculates with the highest concentrations. The highest percentage of morphologically abnormal sperm was noted in ejaculates with low concentrations. Conclusion: The dimensions and shape of sperm depend on the sperm concentration in the ejaculate. In ejaculates with the highest concentrations, the sperm have larger dimensions. Sperm concentration affects the frequency of morphological anomalies in the spermatozoa.
\end{abstract}

Keywords: boar, ejaculate, sperm concentration, sperm morphology.

\section{Introduction}

Male fertility depends on the quality of the spermatozoa produced. Numerous authors have reported that differences in the fertility of males used for artificial insemination (AI) may result from sperm morphology (19). The structure and size of spermatozoa affect their function, including their capacity to undergo the acrosome reaction (20) and to bind to the zona pellucida of the oocyte (6), and thus affect the potential fertility of the male (21). Sperm morphology, however, varies considerably depending on the breed of the male (27) and across different species (29). Variability in sperm morphology is also noted in individuals from the same population (9).

Normal morphological structure guarantees that the spermatozoon will successfully reach the ovum and activate the oocyte (6). Male fertility has been shown to depend on the frequency of morphologically abnormal spermatozoa (1). It has been demonstrated that the presence of abnormal sperm morphology can be the cause of abortion in the initial period of pregnancy (3), reduced embryo quality (17), and decreased fertilisation capacity of sperm (6). Some studies have indicated an association between the dimensions and shape of spermatozoa and male fertility (30), and one cited the size of the sperm head as a factor of male conceptive fitness (23). The dimensions and shape of the sperm cells have also been shown to be affected by the ejaculate traits (9) and environmental factors (10).

Hypor boars, a hybrid line of pigs, are commonly used in the production of hybrid fatteners. While boar sperm quality is routinely assessed by measuring the concentration and morphology of spermatozoa in AI centres, hypor boar sperm morphology specifically has not yet been investigated. It is possible that sperm shape and dimensions depend on the concentration of spermatozoa in the ejaculate. In the present study, an attempt was made to determine the dependence of morphological and morphometric sperm characteristics 
and the ejaculate traits of Hypor boars on sperm concentration in the ejaculate.

\section{Material and Methods}

All boars used in the study were managed under unified conditions, conforming to the current animal welfare regulations. Experiments were conducted using 12 Hypor boars. In total, 120 ejaculates were evaluated, collected at three insemination stations (A, B, and C). Station A is located at latitude $52^{\circ} 52^{\prime} 54^{\prime \prime} \mathrm{N}$ and longitude $20^{\circ} 36^{\prime} 38^{\prime \prime} \mathrm{E}$, station $\mathrm{B}$ at latitude $51^{\circ} 58^{\prime} 27^{\prime \prime} \mathrm{N}$ and longitude $19^{\circ} 01^{\prime} 17^{\prime \prime} \mathrm{E}$, and station $\mathrm{C}$ at latitude $52^{\circ} 05^{\prime} 28^{\prime \prime} \mathrm{N}$ and longitude $19^{\circ} 59^{\prime} 01^{\prime \prime} \mathrm{E}$. The ejaculates were collected using the gloved-hand technique (14) every 4-5 days. At least 10 ejaculates from each boar were collected and assessed. The ejaculates were graded according to sperm concentration in the ejaculate, distinguishing three groups: ejaculates with low sperm concentration (less than $390 \times 10^{6} / \mathrm{mL}, \mathrm{n}-44$ ), ejaculates with medium sperm concentration $\left(390-490 \times 10^{6} / \mathrm{mL}\right.$, $\mathrm{n}-39)$, and ejaculates with high sperm concentration $\left(>490 \times 10^{6} / \mathrm{mL}, \mathrm{n}-37\right)$.

Immediately after obtaining the ejaculate, the following parameters were determined: volume, sperm concentration, total number of spermatozoa, percentage of spermatozoa with progressive motility, and number of insemination doses per ejaculate. Ejaculate volume was determined after isolating the gelatinous fraction on the basis of ejaculate weight measured on an electronic scale. The volume of the ejaculate was calculated assuming a density of $1.0 \mathrm{~g} / \mathrm{mL}$, and sperm concentration in an ejaculate was determined on an AccuRead photometer (IMV Technologies, France). The collected sperm was diluted in Biosolwens Plus (Biochefa, Poland). A blank tube was loaded with $2.4 \mathrm{~mL}$ of $0.9 \% \mathrm{NaCl}$, and a sample tube with $100 \mu \mathrm{L}$ semen sample added to $0.9 \% \mathrm{NaCl}$. Sperm motility was assessed by microscope under $200 \times$ magnification. The total number of spermatozoa, and number of insemination doses per ejaculate were calculated using WINSUL software.

Morphometric measurements and morphological evaluation of the sperm were based on the microscopic examination of slides prepared from fresh ejaculates, using $100 \times$ immersion objectives and a Nikon E-50i light microscope. Morphometric measurements of 15 randomly selected spermatozoa were taken from each slide. The preparations for analyses were made according to the following methodology: a drop of semen was placed on a slide preheated to $37^{\circ} \mathrm{C}$, the slides were allowed to air dry for a minimum of $2 \mathrm{~h}$, and then were prepared and preserved in a $96 \%$ ethanol solution during a 5-minute exposure. After $30 \mathrm{~min}$, the preserved slides were washed in distilled water, and then stained with a $10 \%$ aqueous solution of eosin during a 20- to 60-sec exposure. The stained slides were washed in distilled water again and stained with gentian pigment during a 3- to 5-min exposure. After staining, the slides were washed and dried. The slides were gently rinsed with distilled water for 2 min to remove debris (15).

For each spermatozoon, the following morphometric measurements were made: perimeter, area, length and width of the head, length of the tail, and its total length. On the basis of the results of morphometric measurements, the following indexes of morphological structure of spermatozoa were calculated: head width/head length ratio, head length/total length ratio, head length/flagellum length ratio, flagellum length/total length ratio, perimeter of the head/total length ratio, head area/total length ratio, and head length $\times$ width/total length ratio. In addition, the morphology of 500 spermatozoa, including those with proper morphology as well as with major or minor abnormalities, was evaluated on each slide according to Blom classification (2).

The analysis of variation of semen traits was carried out according to the following model: $\mathrm{Y}_{i j}=\mu+\mathrm{a}_{i}+\mathrm{e}_{i j}$, where $\mathrm{Y}_{i j}$ is trait value, $\mu$ is population mean, $\mathrm{a}_{i}$ is sperm concentration, and $\mathrm{e}_{i j}$ is error. Differences between means were evaluated using Tukey's test at $\mathrm{P} \leq 0.05$.

\section{Results}

Table 1 presents the results of the morphometric measurements of the sperm in relation to the sperm concentration in the ejaculate.

Table 1. Morphometric characteristics of spermatozoa related to sperm concentration in ejaculate (means $\pm \mathrm{SD}$ )

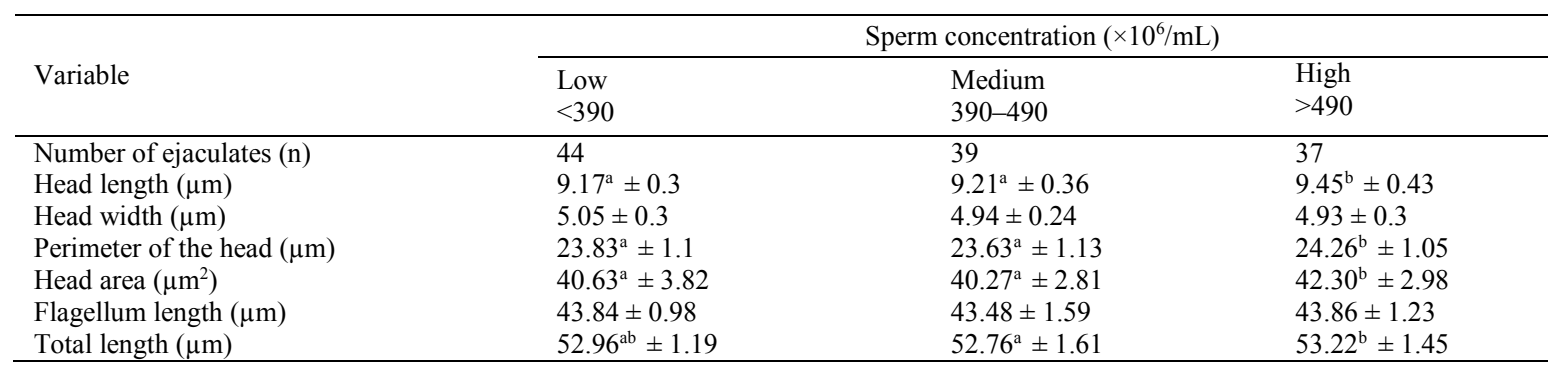

${ }^{\mathrm{a}, \mathrm{b}}$ Differences between average values, represented by different letters in the same row, are significant $(\mathrm{P} \leq 0.05)$ 
The sperm from the ejaculates with a sperm concentration of over $490 \times 10^{6} / \mathrm{mL}$ (high concentration) had larger dimensions than the sperm from ejaculates with medium or low sperm concentration. In the ejaculates with the highest sperm concentration, the sperm heads were $0.24 \mu \mathrm{m}$ longer than those of sperm in ejaculates with medium sperm concentration $(\mathrm{P} \leq 0.05)$ and $0.28 \mu \mathrm{m}$ larger than those of sperm in ejaculates with low sperm concentration $(\mathrm{P} \leq 0.05)$. The sperm from ejaculates with the highest sperm concentration had a larger head perimeter and head area than the sperm from ejaculates with medium or low sperm concentration $(\mathrm{P} \leq 0.05)$. Ejaculates with the highest sperm concentration contained longer sperm than did ejaculates with medium sperm concentration $(\mathrm{P} \leq 0.05)$.

Table 2 presents the indices used to evaluate differences in the shape of sperm from ejaculates with varied sperm concentrations.

The data in Table 2 indicate that as sperm concentration increases, the ratio of head width to head length decreases. In the ejaculates with low sperm concentration, this ratio was 1.43 greater than in the ejaculates with medium sperm concentration, and 2.01 greater than in the ejaculates with high sperm concentration $(\mathrm{P} \leq 0.05)$.
The sperm from ejaculates with the highest sperm concentration had greater ratios of head length to total sperm length and head length to tail length than the sperm from the ejaculates with medium or low sperm concentration $(\mathrm{P} \leq 0.05)$. The sperm from ejaculates with the highest sperm concentration were also shown to have a significantly greater ratio of head area to total sperm length than sperm from ejaculates with medium or low sperm concentration $(\mathrm{P} \leq 0.05)$.

Table 3 presents data on the frequency of morphological sperm anomalies in relation to sperm concentration in the ejaculate.

The data in Table 3 show that as sperm concentration in the ejaculate increases, the frequency of sperm with morphological abnormalities decreases. These tendencies were noted for major defects $(\mathrm{P} \leq 0.05)$, but a slight decrease was also observed in the frequency of minor morphological anomalies. At the same time, as sperm concentration in the ejaculate increased, the percentage of morphologically normal sperm increased as well $(\mathrm{P} \leq 0.05)$.

Table 4 presents data pertaining to the selected semen traits in relation to sperm concentration in the ejaculate.

Table 2. Morphometric indexes of spermatozoa related to sperm concentration in ejaculate (means $\pm \mathrm{SD}$ )

\begin{tabular}{llll}
\hline & \multicolumn{3}{c}{ Sperm concentration $\left(\times 10^{6} / \mathrm{mL}\right)$} \\
\cline { 2 - 4 } Variable (\%) & Low & Medium & $39-490$ \\
\hline Number of ejaculates (n) & $<390$ & 39 & 39 \\
Head width/head length & 44 & $53.61^{\mathrm{a}} \pm 1.92$ & $53.03^{\mathrm{a}} \pm 3.13$ \\
Head length/total length & $55.04^{\mathrm{b}} \pm 2.34$ & $17.47^{\mathrm{ab}} \pm 0.75$ & $17.76^{\mathrm{b}} \pm 0.67$ \\
Head length/flagellum length & $17.33^{\mathrm{a}} \pm 0.52$ & $21.21^{\mathrm{ab}} \pm 1.13$ & $21.58^{\mathrm{b}} \pm 0.96$ \\
Perimeter of the head/total length & $20.93^{\mathrm{a}} \pm 0.7$ & $44.81 \pm 2.1$ & $45.58 \pm 1.31$ \\
Head area/total length & $44.99 \pm 1.8$ & $76.35^{\mathrm{a}} \pm 5.15$ & $79.43^{\mathrm{b}} \pm 4.35$ \\
Head length $\times$ width/total length & $76.3^{\mathrm{a}} \pm 6.63$ & $86.35 \pm 7.22$ & $88.98 \pm 6.67$ \\
Flagellum length/total length & $87.59 \pm 6.93$ & $82.41 \pm 0.98$ & $82.34 \pm 0.8$ \\
\hline
\end{tabular}

${ }^{\mathrm{a}, \mathrm{b}}$ Differences between average values, represented by different letters in the same row, are significant $(\mathrm{P} \leq 0.05)$

Table 3. Frequency of occurrence of morphologically changed spermatozoa related to the sperm concentration in ejaculate (means $\pm \mathrm{SD}$ )

\begin{tabular}{llll}
\hline & \multicolumn{3}{c}{ Sperm concentration $\left(\times 10^{6} / \mathrm{mL}\right)$} \\
\cline { 2 - 4 } Variable (\%) & Low & Medium & High \\
& $<390$ & $390-490$ & $>490$ \\
\hline Number of ejaculates (n) & 44 & 39 & 37 \\
Normal spermatozoa & $94.61^{\mathrm{a}} \pm 3.76$ & $95.69^{\mathrm{ab}} \pm 2.44$ & $96.79^{\mathrm{b}} \pm 2.95$ \\
Sperm with major abnormalities & $1.98^{\mathrm{b}} \pm 2.26$ & $1.18^{\mathrm{ab}} \pm 1.44$ & $0.64^{\mathrm{a}} \pm 0.78$ \\
Sperm with minor abnormalities & $3.42 \pm 2.35$ & $3.13 \pm 2.41$ & $2.58 \pm 2.56$ \\
\hline
\end{tabular}

${ }^{a, b}$ Differences between average values, represented by different letters in the same row, are significant $(\mathrm{P} \leq 0.05)$

Table 4. Basic characteristics of semen traits related to sperm concentration in ejaculate (means \pm SD)

\begin{tabular}{llll}
\hline & \multicolumn{3}{c}{ Sperm concentration $\left(\times 10^{6} / \mathrm{mL}\right)$} \\
\cline { 2 - 4 } Variable & Low & Medium & High \\
& $<390$ & $390-490$ & 390 \\
\hline Number of ejaculates $(\mathrm{n})$ & 44 & $420.00^{\mathrm{b}} \pm 28.67$ & 37 \\
Sperm concentration $\left(\times 10^{6} / \mathrm{mL}\right)$ & $314.38^{\mathrm{a}} \pm 43.39$ & $553.64^{\mathrm{c}} \pm 47.89$ \\
Ejaculate volume $(\mathrm{mL})$ & $344.87^{\mathrm{c}} \pm 90.11$ & $325.53^{\mathrm{b}} \pm 59.85$ & $287.05^{\mathrm{a}} \pm 56.47$ \\
Total number of spermatozoa $\left(\times 10^{9}\right)$ & $118.34 \pm 20.98$ & $109.50 \pm 20.89$ & $98.49 \pm 23.97$ \\
Percentage of spermatozoa with normal motility $(\%)$ & $78.21 \pm 3.89$ & $78.68 \pm 3.43$ & $77.95 \pm 4.08$ \\
Number of insemination doses per ejaculate & $28.67^{\mathrm{a}} \pm 9.26$ & $34.63^{\mathrm{b}} \pm 6.59$ & $40.25^{\mathrm{c}} \pm 7.69$ \\
\hline
\end{tabular}

${ }^{a, b}$ Differences between average values, represented by different letters in the same row, are significant $(\mathrm{P} \leq 0.05)$ 
The data presented in Table 4 show that the volume of ejaculates with low sperm concentration was larger by nearly $20 \mathrm{~mL}$ than that of ejaculates with medium sperm concentration, and by nearly $58 \mathrm{~mL}$ than that of ejaculates with high sperm concentration $(\mathrm{P} \leq 0.05)$. The data also indicate that as sperm concentration increases, the number of insemination doses obtained from the ejaculate increases as well. On average, 5.62 more insemination doses could be prepared from the ejaculates with the highest sperm concentration than from the ejaculates with medium sperm concentration, and 11.58 more than from ejaculates with low sperm concentration $(\mathrm{P} \leq 0.05)$. Sperm motility, on the other hand, was not shown to depend on sperm concentration in the ejaculate.

\section{Discussion}

This study was carried out with the use of the ejaculates of Hypor boars, commonly used in the production of hybrid fatteners. The data presented indicate interrelatedness between the morphological characteristics of sperm and their concentration in the ejaculate. Differences were observed in the dimensions and shape of spermatozoa and in the frequency of the morphological anomalies between ejaculates differing in sperm concentration. The data obtained in the present study show that the length of the sperm heads increases with the sperm concentration. A study by Rijsselaere et al. (25) on dog semen showed that the sperm concentration in dog ejaculates can affect the sperm dimensions. The study showed that sperm from ejaculates with a lower sperm concentration had shorter and narrower heads with a smaller surface area and perimeter than sperm from ejaculates with a higher sperm concentration. The present study shows an analogous dependence of sperm head dimensions on sperm concentration in the ejaculates of domestic pigs. Sperm head dimensions can affect fertility, as variation in sperm head dimensions has been shown to be linked to differences in the cell nucleus chromatin structure (26). Even minor changes in the shape of the sperm head may be accompanied by changes in the chromatin structure in the cell nucleus, which can result in reduced fertility (5).

The dimensions and shape of sperm affect their motility and fertilisation capacity. According to Mossman et al. (22), sperm length is positively correlated with the speed of its movement. Sperm with longer tails are more competitive because they can reach the ovum more quickly (24). The hydrodynamics of the spermatozoon are also affected by the shape of the head. According to Malo et al. (18), sperm with elongated heads move faster than those with rounded heads. Some authors report differences in the intensity and forms of sperm movement depending on the shape of the sperm head (8). Sperm with higher ellipticity values (head length/width) and wider midpieces display a lower capacity for progressive movement in a straight line (7). The present study showed that the ratio of the head dimensions to the tail length of the sperm or to the length of the entire sperm was always the greatest in the sperm from ejaculates with the highest sperm concentration. The ejaculates with the highest sperm concentration had the narrowest and at the same time the most elongated heads. Data obtained by Hirai et al. (12) show that the sperm of boars with lower fertilisation capacity have larger and more elongated heads than the sperm of boars with high fertility rates. An impact of sperm concentration in an ejaculate on their morphometric characteristics has also been reported in stallions. The more concentrated ejaculates were found to contain spermatozoa with smaller and less elongated heads as opposed to the less concentrated ejaculates (4). Sperm dimensions can also be affected by the semen storage (11).

The data of the present study show that the quality of the semen of Hypor boars is very high. The mean percentage of sperm with major morphological defects did not exceed $1.98 \%$. These data show, however, that the frequency of major morphological defects decreases as sperm concentration in the ejaculate increases, while the percentage of morphologically correct sperm increases. In the ejaculates with the highest sperm concentration, over $96.8 \%$ of the sperm were morphologically normal. Studies by Rijsselaere et al. (25) have shown that less concentrated ejaculates contain fewer spermatozoa with morphological alterations, while as the sperm concentration rises, the percentage of morphological anomalies also increases. The frequency of morphological sperm anomalies is influenced by genetic factors and breed of the male, by individual predispositions of the male (16), and by seasonal and environmental factors (10). Sperm deformations included among major defects usually appear during spermatogenesis. The frequency of these anomalies determines the fertility of the male (13). The present study showed that the percentage of sperm cells with minor anomalies was greater than the percentage with major defects, but the frequency of minor sperm defects was also not high. Little influence was felt from the sperm concentration on the frequency of spermatozoa with minor defects, and the differences between the groups were not confirmed statistically. The data in the present study show that sperm concentration in the ejaculate is linked to the quantitative characteristics of the ejaculate. As sperm concentration increases, the ejaculate volume decreases, which was confirmed statistically. These data thus confirm earlier observations that the correlation between sperm concentration and ejaculate volume in boars is inversely proportional (28). Increasing sperm concentration in the ejaculate translated to a marked increase in the number of insemination doses produced from one ejaculate.

To sum up, the dimensions and shape of sperm depend significantly on the sperm concentration in the ejaculate. In ejaculates with the highest sperm 
concentration, the sperm have larger dimensions. Sperm concentration also influences the shape of the sperm, and more precisely, in ejaculates with higher sperm concentration, the sperm heads are more rounded than in the case of ejaculates with a lower sperm concentration. Sperm concentration in addition affects the frequency of morphological anomalies in the sperm, the highest percentage of such was noted in the ejaculates with low sperm concentration.

Conflict of Interests Statement: The authors declare that there is no conflict of interests regarding the publication of this article.

Financial Disclosure Statement: The study was financed by the statutory activity.

Animal Rights Statement: The experiments on animals were conducted in accordance with local Ethical Committee Laws of Siedlce University of Natural Sciences and Humanities and regulations as regards care and use of laboratory animals.

\section{References}

1. Alm K., Peltoniemi O., Koskinen E., Andersson M.: Porcine field fertility with two different insemination doses and the effect of sperm morphology. Reprod Dom Anim 2006, 41, 210-213.

2. Blom E.: The morphological estimation of the spermatozoa defects of bull II: The proposal of new classification of spermatozoa defects. Med Weter 1981, 37, 239-242.

3. Cao X., Cui Y., Zhang X., Lou J., Zhou J., Wei R.: The correlation of sperm morphology with unexplained recurrent spontaneous abortion: a systematic review and meta-analysis. Oncotarget 2017, 8, 55646-55656.

4. Davis R.O., Gravance C.G., Casey P.J.: Automated morphometric analysis of stallion spermatozoa. Am J Vet Res 1993, 11, 1808-1811.

5. Evenson D.P., Wixon R.: Clinical aspects of sperm DNA fragmentation detection and male infertility. Theriogenology 2006, 65, 979-991.

6. García-Vázquez F.A., Gadea J., Matás C., Holt W.V.: Importance of sperm morphology during sperm transport and fertilization in mammals. Asian J Androl 2016, 18, 844-850.

7. Gil M.C., García-Herreros M., Barón F.J., Aparicio I.M., Santos A.J., García-Marín L.J.: Morphometry of porcine spermatozoa and its functional significance in relation with the motility parameters in fresh semen. Theriogenology 2009, 71, 254-263.

8. Gillies E., Cannon R., Green R., Pacey A.: Hydrodynamic propulsion of human sperm. J Fluid Mech 2009, 625, 445-474.

9. Górski K., Kondracki S., Strachocka K., Wysokińska A.: Association of ejaculate sperm counts with their morphological and morphometric characteristics in Hypor boars. Ann Anim Sci 2017, 17, 1043-1052.

10. Górski K., Kondracki S., Wysokińska A.: Effects of season on semen parameters and relationships between selected semen characteristics in Hypor boars. Turk J Vet Anim Sci 2017, 41, 563-569.

11. Hidalgo M., Rodriguez I., Dorado J.M., Soler C.: Morphometric classification of Spanish thoroughbred stallion sperm heads. Anim Reprod Sci 2008, 103, 374-378.
12. Hirai M., Boersma A., Hofflich A., Wolf E., Föll J., Aumüller R., Braun A.J.: Objectively measured sperm motility and sperm head morphometry in boars (Sus scrofa): relation to fertility and seminal plasma growth factors. J Androl 2001, 22, $104-110$

13. Johnson L.A., Weitze K.F., Fiser P., Maxwell W.M.C.: Storage of boar semen. Anim Reprod Sci 2000, 62, 143-172.

14. King G.J., Macpherson J.W.: A comparison of two methods for boar semen collection. J Anim Sci 1973, 36, 563-565.

15. Kondracki S., Banaszewska D., Mielnicka C.: The effect of age on the morphometric sperm traits of domestic pigs. Cell Mol Biol Lett 2005, 10, 3-13.

16. Kondracki S., Wysokińska A., Kania M., Górski K.: Application of two staining methods for sperm morphometric evaluation in domestic pigs. J Vet Res 2017, 61, 345-349.

17. Loutradi K.E., Tarlatzis B.C., Goulis D.G., Zepiridis L., Pagou T., Chatziioannou E., Grimbizis G.F., Papadimas I., Bontis I.: The effects of sperm quality in embryo development after intracytoplasmic sperm injection. J Assist Reprod Gen 2006, 23, 69-74.

18. Malo A.F., Gomendio M., Garde J., Lang-Lenton B., Soler A.J., Roldan E.R.S.: Sperm design and sperm function. Biol Lett 2006, 2, 246-249.

19. Maroto-Morales A., Ramon M., Garcia-Alvarez O., Soler A.J., Esteso M.C., Martinez-Pastor F., Perez-Guzman M.D., Garde J.J.: Characterization of ram (Ovis aries) sperm head morphometry using the Sperm-Class Analyzer. Theriogenology 2010, 73, 437-448.

20. Menkveld R., El-Garem Y., Schill W.B., Henkel R.: Relationship between human sperm morphology and acrosomal function. J Assist Reprod Gen 2003, 20, 432-438.

21. Menkveld R.: Clinical significance of the low normal sperm morphology value as proposed in the $5^{\text {th }}$ WHO laboratory manual for the examination and processing of human semen. Asian J Androl 2010, 12, 47-58.

22. Mossman J., Slate J., Humphries S., Birkhead T.: Sperm morphology and velocity are genetically codetermined in the zebra finch. Evolution 2009, 63, 2730-2737.

23. Pena F.J., Saravia F., Garcia-Herreros M., Nunez-Martinez I., Tapia J.A., Johannisson A., Wallgren M., Rodriguez-Martinez H.: Identification of sperm morphometric subpopulations in two different portions of the boar ejaculate and its relation to post thaw quality. J Androl 2005, 26, 716-723.

24. Pesch S., Bergmann M.: Structure of mammalian spermatozoa in respect to viability, fertility, and cryopreservation. Micron 2006, 37, 597-612.

25. Rijsselaere T., Soom A., Hoflack G., Meas D., Kruif A.: Automated sperm morphometry and morphology analysis of canine semen by the Hamilton-Thorne analyser. Theriogenology 2004, 62, 1292-1306.

26. Sailer B.L., Jost L.K., Evenson D.P.: Bull sperm head morphometry related to abnormal chromatin structure and fertility. Cytometry 1996, 24, 167-173.

27. Saravia F., Nunez-Martinez I., Moran J.M., Soler C., Muriel A., Rodriguez-Martinez H., Pena F.J.: Differences in boar sperm head shape and dimensions recorded by computer-assisted sperm morphometry are not related to chromatin integrity. Theriogenology 2007, 68, 196-203.

28. Smital J.: Effects influencing boar semen. Anim Reprod Sci 2009, $110,335-346$

29. Sousa P.C., Santos E.A.A., Souza A.L.P., Lima G.L., Barros F.F.P.C., Oliveira M.F., Silva A.R.: Sperm morphological and morphometric evaluation in captive collared peccaries (Pecari tajacu). Pesq Vet Bras 2013, 33, 924-930.

30. Sukcharoen N., Sithipravej T., Promvienghai S., Chinpilas V., Boomkasemsanti W.: Sperm morphology evaluated by computer (IVOS) cannot predict the fertilization rate in vitro after intracytoplasmic sperm injection. Fertil Steril 1998, 69, 564-568. 